Research Article

\title{
Modelling and Control of Proposed Two Dodecacopter Systems
}

\author{
Şahin Yıldırım $^{a} *$ (D), Nihat Çabuk ${ }^{b}$ (D), Veli Bakırcıoğlu ${ }^{b}$ \\ ${ }^{a}$ Mechatronic Engineering Department, Faculty of Engineering, Erciyes University, Kayseri, Turkey \\ ${ }^{b}$ Aksaray University, Technical science Vocational school,Aksaray, Turkey
}

\begin{tabular}{ll}
\hline ARTICLE INFO & ABSTRACT \\
\cline { 3 - 3 } $\begin{array}{l}\text { Article history: } \\
\text { Received 12 March 2020 }\end{array}$ & $\begin{array}{l}\text { Nowadays, the use of multi-propellers has been increased, because of some basic advantages such } \\
\text { as vertical take-off and landing, simple mechanical structure. In addition, different usage fields }\end{array}$ \\
Accepted 17 April 2020 & such as carrying an object and making video records over the cities. This paper exposes design \\
Keywords: & and control of two different multi propeller systems and both systems have 12 rotors to carry more \\
Design & load. In first, all motors are arranged at identical angles in the same plane. In the other, the motors \\
Modelling & are mounted in two groups, eight and four in two different planes, the lower and upper. Flight \\
Multirotor UAV & performances of these two different UAVs were compared under disturbing effect. It was observed \\
Simulation & that the flight performances were close in the simulation study. Although the average and \\
& maximum of the position errors for three axes in the first model was less than the other, for the \\
& same propeller dimensions, the first model must have larger dimensions than the other in the \\
& context of the smallest vehicle dimensions.
\end{tabular}

This is an open access article under the CC BY-SA 4.0 license. (https://creativecommons.org/licenses/by-sa/4.0/)

\section{Introduction}

Unmanned Aerial Vehicles (UAVs) have recently aroused great interests in both industrial and military fields. UAV is a type of very complex system which integrates different hardware components, such as camera, Global Positioning System (GPS), Inertial Management Unit (IMU), controller, and different software components, such as image processing, path planning and inner loop control [1]. Due to the ability to perform dangerous and repetitive tasks in remote and hazardous environments, UAV is very promising to play more important roles in many applications and recent developments have proven the benefits in different ways [2].

Multirotor UAVs are capable of take-off and landing vertically (VTOL). Six degree-of-freedom are obtained by changing the angular speed of the motors of these vehicles. The number of motor variable from three and eight standards on the basis of these according to the usage of the vehicle are determined.

Brushless DC motors [3] are mostly used in these vehicles. These motors derive their energy from lithium polymer (Li-PO) batteries [4]. The energy requirement of the vehicle in consideration when determining the total weight of the vehicle, the weight of the vehicle motor and also the selection of the proper propeller is one of the important issues in the design. Regardless of the weight of the vehicle, a controller card and software which will be used to control the vehicle in the desired manner requires sensors that help to fly of the vehicle.

To measure roll, pitch and yaw positions, velocities and accelerations is used a gyro sensor, to measure height of the vehicle is used a pressure sensor, to determine of direction of the vehicle is used compass, to measure the global position of the vehicle is used a GPS. Especially the GPS and compass sensors are able to basically be affected by the magnetic field of the vehicle in the calibration and use of the vehicle because this point in time are taken into consideration. Used to drive motors, each motor suitable for electronic speed controller (ESC) is one of the important components of. The motors which supply power to the ESC and wires and connection cables since it may be exposed to high temperatures that will be used should be selected in consideration of this point solders.

Mechanically the vehicle is designed to consider the

* Corresponding author. E-mail address: sahiny@erciyes.edu.tr DOI: $10.18100 /$ ijamec.698462 
total weight of the vehicle when one of the important issues. In the mechanical structure, the main consideration is durability and lightness. For this reason, the material to be used should be chosen accordingly. The physical design and control of multirotor air vehicle have studied in the literature [5-16]. Taehyung Kim, and sung-Kyung Hong [2], multicopter hybrid design study, two internal combustion engine the center of gravity multicopter, brushless DC motors have developed through the four corners of a four-Assembly model. As a result of the application of this model expressed that they get more flight time.

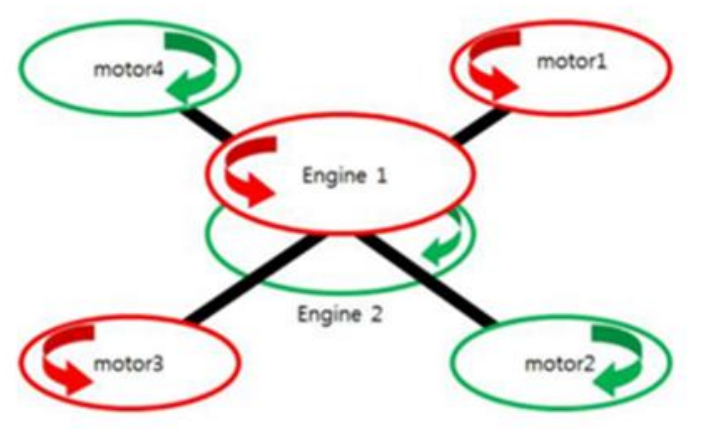

Figure 1. Hybrid multi propeller model [2]

China E. Lin et al. [17], developed a hybrid vehicle model by installing four short-arm internal combustion engines and four long-arm dc motors. Longer flight times and greater load capacity are made to enable the goals of this study stated that they have achieved.

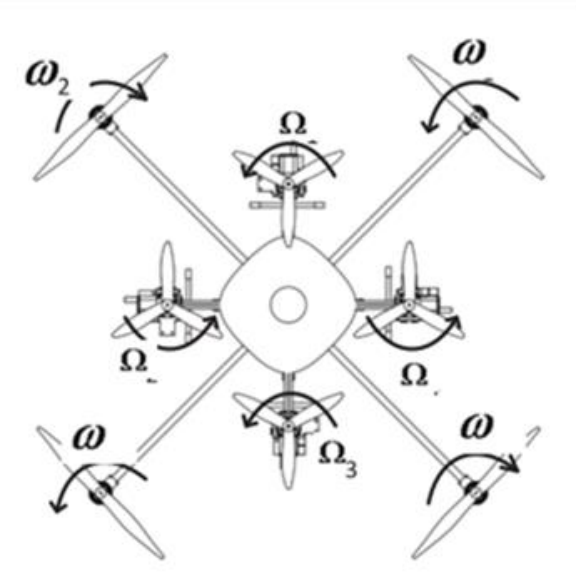

Figure 2. Other type multi propeller model [17]

J. Verbeke et al. [1], said that we have conceived a novel compound multicopter configuration specifically for flight through narrow corridors, its design combines the contradictory requirements of limited width, high agility and long endurance while carrying a significant payload.

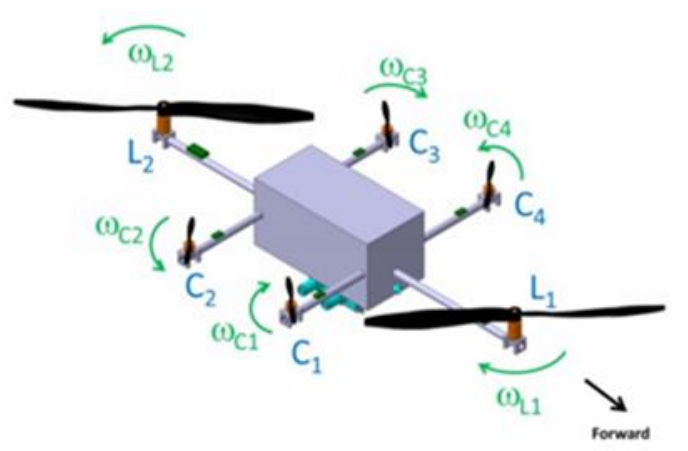

Figure 3. Model of multicopter with different wind [1]

In this study, two different types of dodecacopter models which are non-traditional structure were developed and simulated for the purpose of carrying loads. The general mathematical model of multi-rotor UAVs can be examined from our previous study [18], as in many publications $[19,20]$.

\section{Design and modelling}

Multi propeller systems begin to design various mechanical systems required for electrical, electronic and mechanical components of the supply phase. These design updates will be made according to changes which may occur in mechanical components. SolidWorks is used to mechanical design and MATLAB program is used to analysis as a simulation program.

In this study, for high lifting capacity, two different types of multicopter air vehicle models with 12 rotors have been proposed. The motors in these models was selected as one of the reasons why the high number of a motor or drive the vehicle in the event of some malfunction of the balance of the ability to make easy and to ensure a safe landing. In this situation it is necessary to develop a suitable control algorithm.

In the first model as seen fig. 4, 12 motors which are independently controlled are arranged at identical angles in the same plane.

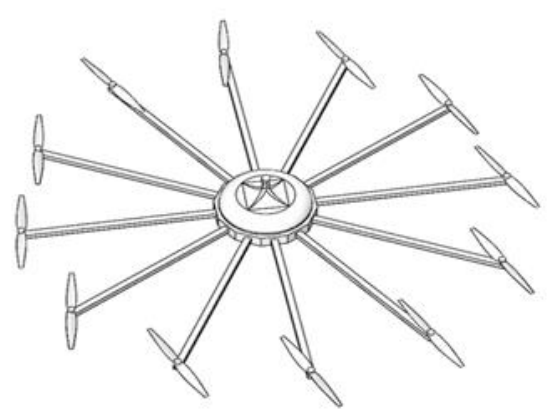

Figure 4. Proposed 12 dodecacopter model

In the second model, smaller sizes to achieve higher lifting capacity is eight in the upper plane of the motors while the four motors are placed on the lower plane and $8+4$ dodecacopter model was created as seen in fig. 5 . 


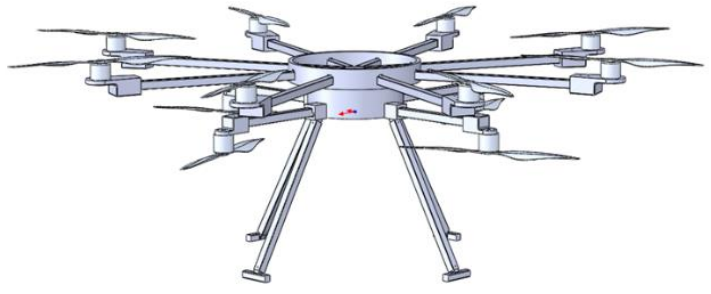

Figure 5. Proposed $8+4$ dodecacopter model

The motors are mounted on the vehicle, which is planned to be planned to be used for motor and propeller, which will occur according to the thrust values for the example are given in Table 1 below. This table KDE4215XF KDE Direct motor with a value of $465 \mathrm{Kv}$ which is planned to be used in this study model of a DC motor belongs to.

Table 1. A proposed motor and propeller thrust values

\begin{tabular}{|c|c|c|c|c|}
\hline $\begin{array}{c}\text { Throttle } \\
\text { Range } \\
{[\%]}\end{array}$ & $\begin{array}{c}\text { Current } \\
{[\mathbf{A}]}\end{array}$ & $\begin{array}{c}\text { Power } \\
{[\mathbf{W}]}\end{array}$ & $\begin{array}{c}\text { Velocity } \\
{[\mathbf{R P M}]}\end{array}$ & $\begin{array}{c}\text { Thrust } \\
{[\mathbf{g}]}\end{array}$ \\
\hline 25 & 2.4 & 62 & 3360 & 620 \\
\hline 50 & 8.5 & 221 & 5340 & 1670 \\
\hline 75 & 23.2 & 605 & 7500 & 3340 \\
\hline 100 & 45.2 & 1179 & 9300 & 5360 \\
\hline
\end{tabular}

According to this table, the most relevant in the period 15.5-inch motor with a propeller of high thrust $5360 \mathrm{~g}$ value in the scale value can be obtained. The weight of the vehicle, which is one of the most important parameters in the selection of take-off have a direct effect on the motor and propeller should be considered. Each of the propeller and the brushless DC motor manufacturers are served by the appropriate table according to. The maximum approximate thrust value for both vehicle models is $64 \mathrm{~kg}$ according to table 1 .

The mathematical model was obtained for the dodecacopter system. Relationship between angular velocities of the motors and the body forces can be formed by a matrix $C$ as in Eq. (1), and angular velocities of the dodecacopter system defined by a vector $\Omega$ in Eq. (2). The desired thrust forces and the moments are indicated by the $U_{d}$ vector.

$$
\begin{aligned}
& U_{d}=C * \Omega^{2} \\
& \Omega^{2}=\left[\begin{array}{lllllllllllllll}
\Omega_{1}{ }^{2} & \Omega_{2}{ }^{2} & \Omega_{3}{ }^{2} & \Omega_{4}{ }^{2} & \Omega_{5}{ }^{2} & \Omega_{6}{ }^{2} & \Omega_{7}{ }^{2} & \Omega_{8}{ }^{2} & \Omega_{9}{ }^{2} & \Omega_{10}{ }^{2} & \Omega_{11}{ }^{2} & \Omega_{12}{ }^{2}
\end{array}\right]^{T}
\end{aligned}
$$

The vector $U_{d}$ is given as Eq. (3), which includes the needed forces and moments for the motion.

$$
\boldsymbol{U}_{\boldsymbol{d}}=\left[\begin{array}{c}
F_{t} \\
M_{r} \\
M_{p} \\
M_{y}
\end{array}\right]
$$

The matrix $C_{1}$ which is for first model is constant and it can be calculated according to the first vehicle's configuration as in Eq. (4).

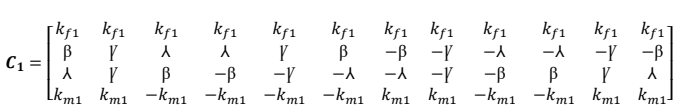

The matrix $C_{2}$ which is for second model is constant and it can be calculated according to the vehicle's configuration as in Eq. (5).

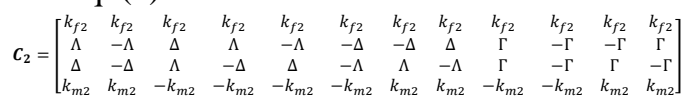

For the sake of simplicity of the matrixes, $\beta, \gamma, \Lambda, \Delta$ and $\Gamma$ given in Eq. (6)

$$
\begin{aligned}
& \beta=k_{f 1} * l_{1} * \sin (15) \\
& Y=k_{f 1} * l_{1} * \sin (45) \\
& \Lambda=k_{f 1} * l_{1} * \sin (75) \\
& \Lambda=k_{f 2} * l_{u} * \sin (22.5) \\
& \Delta=k_{f 2} * l_{u} * \sin (67.5) \\
& \Gamma=k_{f 2} * l_{d} * \sin (45)
\end{aligned}
$$

where, $k_{f 1}$ and $k_{f 2}$ are thrust factors, $k_{m 1}$ and $k_{m 2}$ reverse moment factors for first and second model, respectively. These factors are obtained experimentally. Besides, $l_{\mathrm{u}}$ and $l_{\mathrm{d}}$ arm length in upper plane and lower plane of second model, respectively. Then, $l_{1}$ is arm length for first model. The $F_{t}$ is the desired force that acts on the body and $M_{r}$ and $M_{p}$ are desired roll and pitch moments. $M_{y}$ is yaw moment, which is the sum of the reaction moments caused by each rotor with propeller.

\section{Simulation Studies}

Simulations were carried out with the prescribed trajectory of the proposed both multi propeller models design.

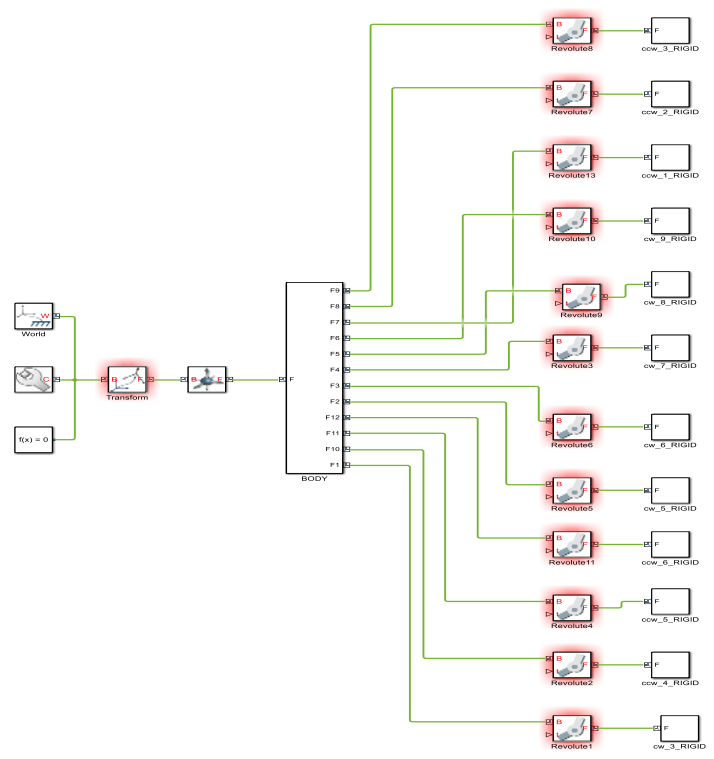

Figure 6. Simulink model of the multi-propeller air vehicles

As shown in figure 6, the Simulink model is obtained to simulate by transferring the 3-D models of the vehicles obtained by a CAD program to MATLAB with their all physical parameters. 
Trajectory of the proposed system was controlled by using standard PID controller with constant gain parameters. However, gain parameters of the controller were tuned with empirically. The systems were modelled and controlled by MATLAB Simulink model as seen in Figure 7.

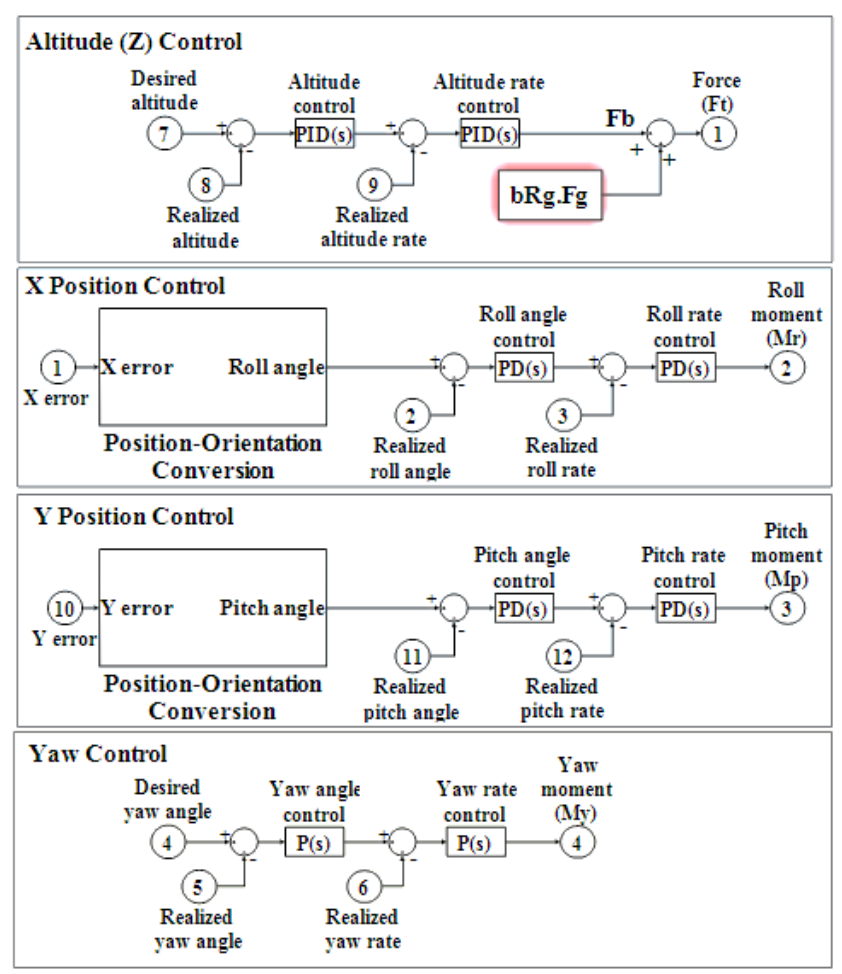

Figure 7. The proposed system control block diagram

Linear speed is limited to $3 \mathrm{~m} / \mathrm{s}$ and maximum tilt angle is 45 degree of both UAV.

The simulation performed under the effect of disturbance force for three axes given in Figure 8. This force represents the effect of winds on the vehicle.

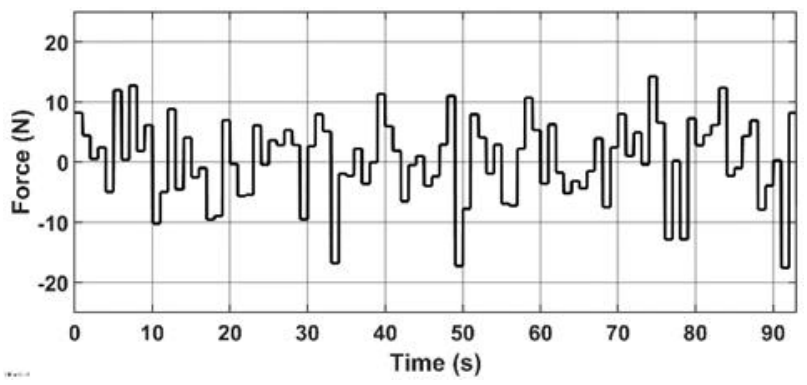

Figure 8. Disturbance effect

As can be seen from Figure 9 and Figure 10, the result of trajectory tracking simulation on three axes is obtained graphically for both models. The average of the root mean square (RMS) values of the position errors occurring in all three axes in the simulation were calculated as $0.915 \mathrm{~m}$ and $1.120 \mathrm{~m}$ for first and second model, respectively.
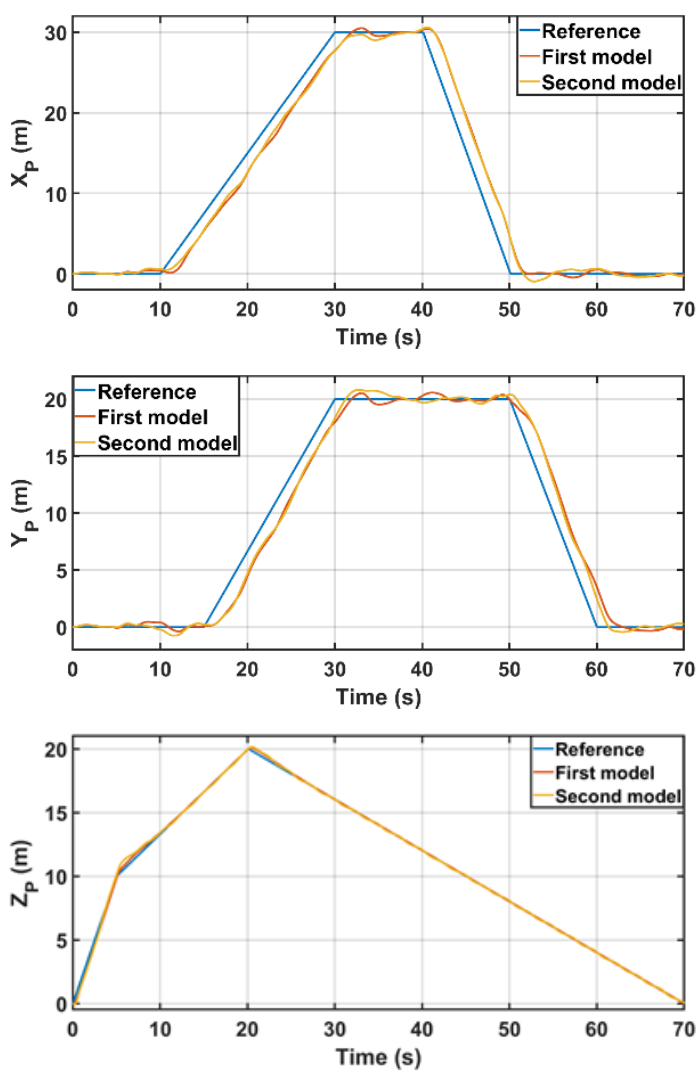

Figure 9. $\mathrm{X}, \mathrm{Y}$ and $\mathrm{Z}$ axes tracking of dodecacopter models

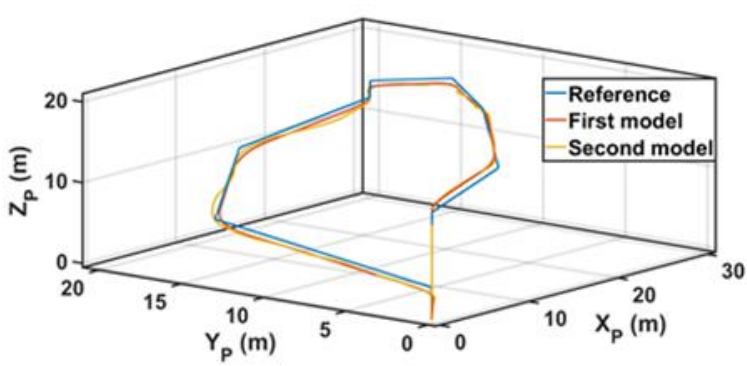

Figure 10. 3-D trajectory tracking of both model

Besides, the maximum and rms values of the position errors occurring in three axes are given in Table 2. At the same time, mean values of the position errors are given in the same table.

Table 2. Numerical results of both model

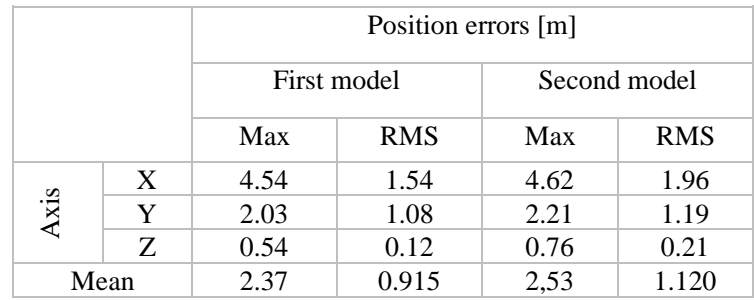

\section{Conclusion}

This study is based on the modeling and simulation of two different twelve rotor UAV with non-traditional 
configurations. The two different UAV models with 12 motors have been proposed for high load carrying capacity. While the motors of the first of these two different models are placed in the same plane, the second model's motors are divided into two separate planes, upper and lower. The simulation study performed in MATLAB environment for both models, PID controllers with the same parameters were used.

According to the results, the trajectory tracking performances in terms of position errors of both models were close, while the error in the second model was found to be less than the other. The results were found satisfactory.

Since the angle between the rotors in the first model is narrower, if both models use the same size propeller, the arm length of the first model must be more. This can be said as a negative situation, since the size of the first model will require more than the second.

This proposed two different type multi-rotor air vehicle model could be utilized in areas where high lifting capacity is important. At the same time, it is thought that it can give an idea to different UAV designs.

\section{Acknowledgments}

This study is supported by Erciyes University Scientific Research Projects Unit within the scope of FBA-20177393 project.

\section{References}

[1] J. Verbeke, D. Hulens, H. Ramon, T. Goedeme, and J. De Schutter, "The design and construction of a high endurance hexacopter suited for narrow corridors," 2014 Int. Conf. Unmanned Aircr. Syst. ICUAS 2014 - Conf. Proc., pp. 543-551, 2014.

[2] T. Kim and S. Hong, "Control System Design and Experimental Validation of Hybrid Multicopter for Endurance Enhancement," Asia-pacific J. Model. Simul. Mech. Syst. Des. Anal., vol. 2, no. 1, pp. 15-20, 2017.

[3] K. Orman, K. Can, A. Başcı, and A. Derdiyok, "Applied Mathematics, Electronics and Computers Real-Time Speed Control of BLDC Motor Based On Fractional Sliding Mode Controller," vol. 4, pp. 314-318, 2016.

[4] Tom, "The 9 Best Lipo Batteries for RC Cars or Quadcopter in 2019 - Reviews," 2018. [Online]. Available: https://rctopgeek.com/best-lipo-battery/. [Accessed: 02-Apr2019].

[5] J. M. Selfridge and G. Tao, "A multivariable adaptive controller for a quadrotor with guaranteed matching conditions," Syst. Sci. Control Eng., vol. 2, no. 1, pp. 24-33, 2014.

[6] X. Shao, J. Liu, H. Cao, C. Shen, and H. Wang, "Robust dynamic surface trajectory tracking control for a quadrotor UAV via extended state observer," Int. J. Robust Nonlinear Control, 2018.

[7] M. Bangura and R. Mahony, "Thrust Control for Multirotor Aerial Vehicles," IEEE Trans. Robot., vol. 33, no. 2, pp. 390405, 2017.

[8] W. Ong, S. Srigrarom, and H. Hesse, "Design methodology for heavy-lift unmanned aerial vehicles with coaxial rotors," AIAA Scitech 2019 Forum, vol. 9781624105, no. January, pp. 7-11, 2019.

[9] M. Hassanalian and A. Abdelkefi, "Classifications, applications, and design challenges of drones: A review," Prog. Aerosp. Sci., vol. 91, no. May, pp. 99-131, May 2017.
[10] S. Zabunov and G. Mardirossian, "Innovative Dodecacopter Design - Bulgarian Knight,” Int. J. Aviat. Aeronaut. Aerosp., vol. 5 , no. $4,2018$.

[11] A. Modirrousta and M. Khodabandeh, "A novel nonlinear hybrid controller design for an uncertain quadrotor with disturbances," Aerosp. Sci. Technol., vol. 45, pp. 294-308, Sep. 2015.

[12] T. Oktay and O. Köse, "Farkli Uçuş Durumlari Için Quadcopter Dinamik Modeli ve Simulasyonu," Eur. J. Sci. Technol., no. 15, pp. 132-142, 2019.

[13] J. J. Sofonia, S. Phinn, C. Roelfsema, F. Kendoul, and Y. Rist, "Modelling the effects of fundamental UAV flight parameters on LiDAR point clouds to facilitate objectives-based planning," ISPRS J. Photogramm. Remote Sens., vol. 149, no. August 2018, pp. 105-118, 2019.

[14] G.-X. Du and Q. Quan, "Optimization of Multicopter Propulsion System Based on Degree of Controllability," $J$. Aircr., vol. 56, no. 5, pp. 2062-2069, 2019.

[15] B. P. De Carvalho, "A Framework for Energy Efficient UAV Trajectory Planning," Concordia University, 2018.

[16] H. Xiu, T. Xu, A. H. Jones, G. Wei, and L. Ren, "A reconfigurable quadcopter with foldable rotor arms and a deployable carrier," in 2017 IEEE International Conference on Robotics and Biomimetics (ROBIO), 2017, vol. 2018-Janua, pp. 1412-1417.

[17] C. E. Lin et al., "Engine controller for hybrid powered dual quad-rotor system," in IECON 2015 - 41 st Annual Conference of the IEEE Industrial Electronics Society, 2015, pp. $001513-$ 001517.

[18] Ş. Yıldırım, N. Çabuk, and V. Bakırcıoğlu, "Design and trajectory control of universal drone system," Measurement, vol. 147, p. 106834, Dec. 2019.

[19] E. Kuantama, R. Tarca, S. Dzitac, I. Dzitac, T. Vesselenyi, and I. Tarca, "The Design and Experimental Development of Air Scanning Using a Sniffer Quadcopter," Sensors, vol. 19, no. 18, p. 3849, Sep. 2019.

[20] A. Chovancová, T. Fico, E. Chovanec, and P. Hubinský, "Mathematical modelling and parameter identification of quadrotor (a survey)," Procedia Eng., vol. 96, pp. 172-181, 2014. 